Check for updates

Cite this: RSC Adv., 2017, 7, 49320

Received 29th July 2017

Accepted 27th September 2017

DOI: $10.1039 / \mathrm{c} 7 \mathrm{ra0} 8385 \mathrm{~h}$

rsc.li/rsc-advances

\section{Preparation and evaluation of polyampholyte inhibitor DAM}

\begin{abstract}
Fengjie $\mathrm{Ma},{ }^{a}$ Xiaolin $\mathrm{Pu},{ }^{* a}$ Bo Wang, (D) ${ }^{\mathrm{b}} \mathrm{Jia} \mathrm{Li}^{\mathrm{a}}$ and Cheng Cao ${ }^{\mathrm{a}}$
In this study, polyampholyte inhibitor DAM was synthesized from three monomers, namely, diallyl dimethyl ammonium chloride (DMDAAC), methyl allyl alcohol (MP) and 2-acrylamido-2-methyl propane sulfonic acid (AMPS), by aqueous solution polymerization. The inhibition performance of DAM was evaluated from the perspectives of particle-size distribution, hot-rolling dispersion test, linear swelling test, and inherent viscosity. Inhibitory mechanisms were investigated by molecular weight pattern analysis, infrared radiation (IR) spectroscopy, zeta potential measurement, thermogravimetric analysis (TGA), and the scanning electron microscope (SEM) technique. The results demonstrated the good inhibitive effect exerted by DAM on the hydration and dispersion of shale, which achieved optimum values and showed a good synergetic effect with the addition of $3 \% \mathrm{KCL}$. Moreover, it is thermally stable at $288^{\circ} \mathrm{C}$.
\end{abstract}

\section{Introduction}

It is estimated that $90 \%$ of wellbore instability problems occur in the shale strata. ${ }^{1}$ The uptake of water in shale weakens the wellbore stability and causes complex issues such as collapsing holes, tight holes, balling bits, and stuck pipes. ${ }^{2,3}$ In order to avoid these problems, oil-based drilling fluids with little or no water are expected to play a role. However, water-based drillings fluids are dominant in horizontal wells drilled in shales due to their advantages over oil-based ones in terms of environmental friendliness and cost. ${ }^{4-6}$ Efforts have been directed to address the welling inhibition problems in shales, which are mostly caused by water.

The negative charge on the clay mineral surface can be reduced by cation exchange with inorganic salt, which can compress the thickness of diffusive electric double layers so as to inhibit shale hydration. ${ }^{7,8}$ However, when there are fewer cations for exchange or the inorganic salt concentration is low, such compression and inhibition effects are not satisfactory. In this context, the addition of a surfactant can reduce the hydrophilicity of the rock surface, thus reducing the wateradsorption capacity of shales and preventing hydration. ${ }^{9}$ Polyalcohol and asphalt surfactants can form hydrophobic layers on the rock surface by plugging the pores of shales so as to slow the migration of filtrates into shales and thus prevent shale hydration. ${ }^{10}$ However, the apertures of pores in shales are on the scale of a few dozen nanometers, making effective blocking difficult. The amino group is known to have a strong adsorption capacity for clay minerals, and various types of cationic organic

${ }^{a}$ State Key Laboratory of Oil and Gas Reservoir Geology and Exploitation, Researching on Drilling Fluid Technology, College of Petroleum Engineering, Southwest Petroleum University, Chengdu, 610500, China.E-mail: mafengjie0906@sohu.com

${ }^{b}$ Research Institute of Yan Chang Petroleum (Group) CO. LTD., Xi'An, Shan Xi, China amine salts have been tried in the field. However, they decompose minerals and produce an odor of ammonia, and they are ineffective in high-temperature conditions. ${ }^{\mathbf{1 1}}$ Studies on other cationic shale inhibitors reveal that high molecular weight has a significant adverse effect on shale inhibition performance. ${ }^{\mathbf{1 2 - 1 4}}$ These inhibitors have various limitations and do not always succeed in inhibiting the hydration of highly water-sensitive clays. Therefore, it is essential and urgent to develop a novel inhibitor featuring an effective inhibition function and environmental friendliness, which is favored in economically viable drilling operations.

Molecular chains of polyampholyte carry both anionic and cationic groups. Previous studies focused on protein macromolecules in amphoteric polymers, among which amino acids, derived from protein hydrolysis and containing both $-\mathrm{NH}_{2}$ and - $\mathrm{COOH}$, were first introduced to inhibitors by Bruton. ${ }^{15}$ Polyampholyte inhibitors feature the stronger adsorption ability of negatively charged clay and better rheological properties and higher filtration capacity than conventional cationic polymer inhibitors. Therefore, more and more attention has been paid to polyampholyte polymers and their performance as drilling fluid inhibitors.

In order to deal with the shale hydration problem caused by water-based drilling fluids, we designed and synthesized a new type of low molecular weight polyampholyte inhibitor DAM, whose performance was then comprehensively evaluated. Strong adsorbent ammonium groups were introduced into molecules, which enable an anion hydrating group $-\mathrm{SO}_{3} \mathrm{Na}$ to bind with an adsorbent non-ionic hydrated group-OH, forming low molecular weight linear polymers. Finally, the synthesized polyampholyte inhibitor was characterized and evaluated, the results of which indicated an excellent inhibitive ability and strong resistance to temperature. 


\section{Materials and methods}

\section{Materials}

Diallyl dimethyl ammonium chloride (DMDAAC) was provided by Shanghai Aladdin Bio-Chem Technology Co., Ltd, China. Methyl allyl alcohol (MP) was obtained from Hubei Ju Sheng Technology Co., Ltd. 2-Acrylamido-2-methyl propane sulfonic acid (AMPS), ammonium persulfate, sodium hydroxide, anhydrous ethanol, and anhydrous methanol were purchased from Chengdu Kelon Chemical Reagent Factory.

\section{Experimental methods}

Synthesis of polyampholyte inhibitor DAM. The synthesis of DAM is based upon the thermal copolymerization method reported by M. Scholl ${ }^{16}$ and S. W. Fox, ${ }^{17}$ with some necessary modifications. Extensive investigations finally determined the aqueous solution method as the polymerization method and ammonium persulfate as the initiator. We performed a series of experiments to find the optimized composition, and the preparation followed the procedures below. A certain amount of AMPS was dissolved in deionized water and the solution was neutralized to $\mathrm{pH}=7$ with $\mathrm{NaOH}$. A 30\% concentration of monomer was added to a three-necked flask with a reflux device, with the molar ratio of AMPS : DMDAAC : MP = 4:3:4. Then, the mixture was stirred and nitrogen gas was kept flowing into the flask for 20 minutes to remove oxygen. When the temperature was raised to $65{ }^{\circ} \mathrm{C}, 4 \%$ ammonium persulfate initiator was added and after a 7 hour period the reaction was stopped and cooled down. After adding anhydrous ethanol to dissolve impurities, a white material was obtained, which was sheared and granulated, and then dried and abraded to a white powder. The reaction equation is shown in Fig. 1.

Hot-rolling dispersion test. This test aimed to mimic the exposure of drilled cuttings to a particular drilling fluid during transportation to the surface through a well bore annulus, ${ }^{\mathbf{1 8}}$ and the dispersion tendency of the shale sample was measured after
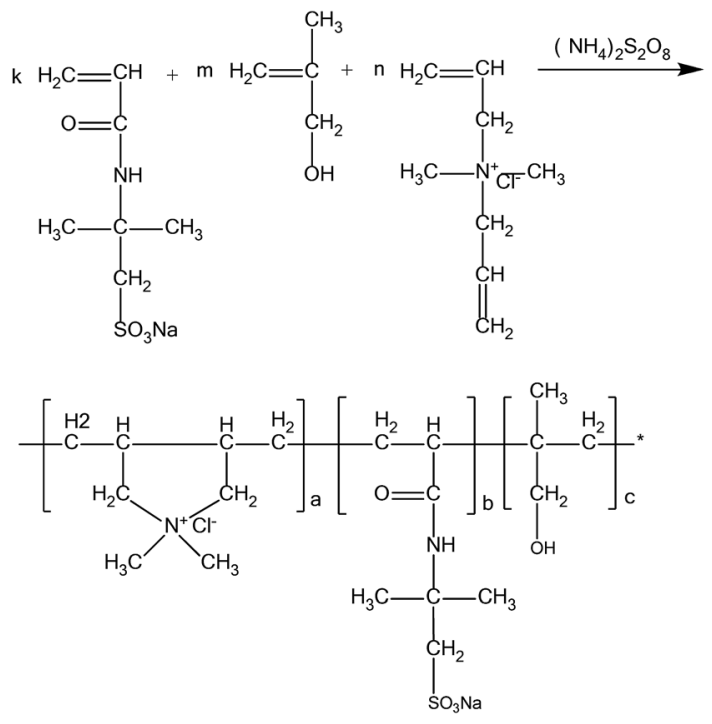

Fig. 1 Ternary copolymer reaction equation. being exposed to a drilling fluid. ${ }^{19} 350 \mathrm{~mL}$ of inhibitor solution at a certain concentration and $50 \mathrm{~g}$ of shale cuttings with sizes of 2-5 mm were contained in a sealed cell and hot-rolled at a certain temperature for 16 hours. After cooling to ambient temperature, remnant shale samples were recovered with a 40 mesh sieve, washed with fresh water, and dried at $105{ }^{\circ} \mathrm{C}$ for 4 hours. Then, the recovered shale cuttings were weighed and calculated to determine the recovery, high values of which generally imply high-quality inhibition.

Linear swelling test. $40 \mathrm{~g}$ of Na-Bent was dispersed in $500 \mathrm{~mL}$ of deionized water and incubated for 24 hours, and then $8 \mathrm{~g}$ of shale inhibitors were added. After stirring vigorously for $30 \mathrm{~min}$, the dispersion was incubated for 24 hours to obtain adsorption equilibrium. Then the dispersions were centrifuged at $8000 \mathrm{rpm}$ for $20 \mathrm{~min}$ and washed with deionized water three times. The sedimentation was dried at $105{ }^{\circ} \mathrm{C}$ and ground to a 200-mesh fine powder. The bentonite was immersed in deionized water for 24 hours, and then centrifuged, dried, and ground to a fine powder for a later linear swelling test.

The linear swelling test was designed to evaluate the swelling rate/ratio of shale samples after contact with the test fluid. ${ }^{20}$ First, $10 \mathrm{~g}$ of Na-Bent was compressed under a pressure of $10 \mathrm{MPa}$ for $5 \mathrm{~min}$ to form a pellet with a diameter of $25.4 \mathrm{~mm}$. Then the shale pellet was put into the shale chamber, which was gently filled with test fluids. Once the shale pellet had made contact with the test fluid, the pellet would adsorb water and swell along the vertical direction. The swelling height was recorded by a sensing device, and the percentage swelling was calculated. A high swelling rate indicated a high potential for hydration and swelling.

Thermogravimetric analysis (TGA). TGA tests were performed on a TGA/DSC1 thermal analyzer from METTLERTOLEDO Inc. (Schwerzenbach, Switzerland) with a heating program from 50 to $1000{ }^{\circ} \mathrm{C}$ at a heating rate of $10 \mathrm{~K} \mathrm{~min}^{-1}$ in a nitrogen flow of $50 \mathrm{~mL} \mathrm{~min}^{-1}$.

Inherent viscosity test. The inherent viscosity of the watersoluble polymer corresponds to its viscosity-average molecular weight. The equipment required for the measurement and operation is simple, but the experimental accuracy is high. ${ }^{21}$ The relationship between the polymer viscosity-average molecular weight and its intrinsic viscosity satisfies the Mak-Houwink equation, as shown in eqn (1).

$$
[\eta]=K M^{\alpha}
$$

$[\eta]$-inherent viscosity, $\mathrm{mL} \mathrm{g}^{-1} ; K$-empirical constants, $\mathrm{mL} \mathrm{g}^{-1}$; $M$-viscosity-average molecular weight; $\alpha$-empirical constant related to molecular shape.

This method refers to the People's Republic of China national standard GB12005.1-89 "Polyacrylamide inherent viscosity determination method", and a single element can be tested for polymer intrinsic viscosity. The formulas are as follows ((2) and (3))

$$
[\eta]=\frac{\sqrt{\frac{2-\ln 3}{\eta_{\mathrm{r}}-\ln \eta_{\mathrm{r}}-1}\left(\eta_{\mathrm{r}}-1\right)}-2 \sqrt{\frac{\eta_{\mathrm{r}}-\ln \eta_{\mathrm{r}}-1}{2-\ln 3}}}{c\left(\sqrt{\frac{2-\ln 3}{\eta_{\mathrm{r}}-\ln \eta_{\mathrm{r}}-1}}-1\right)}
$$


Table 1 Effect of DAM on the particle-size distribution of bentonite dispersion

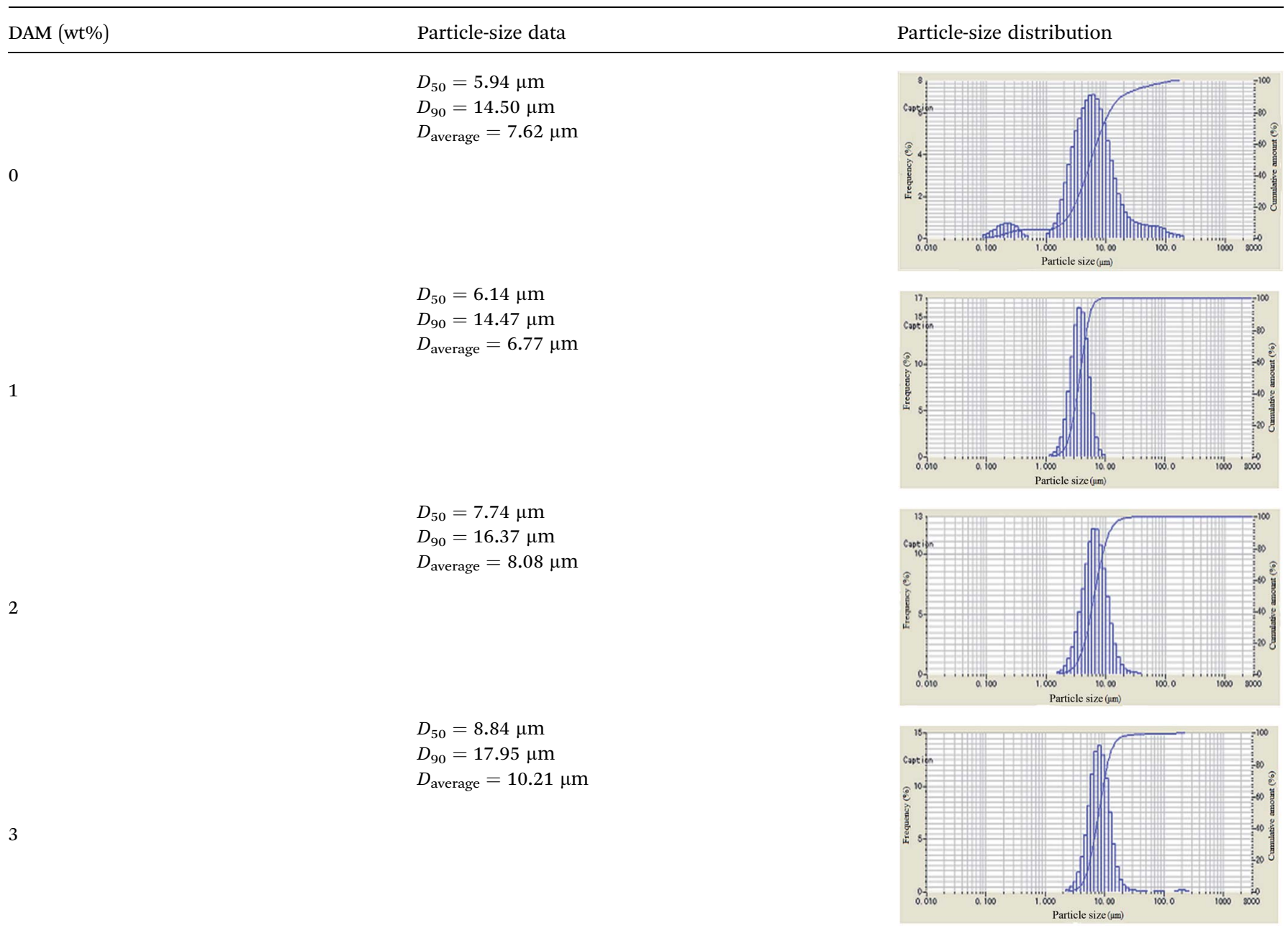

$$
\begin{aligned}
& D_{50}=6.14 \mu \mathrm{m} \\
& D_{90}=14.47 \mu \mathrm{m} \\
& D_{\text {average }}=6.77 \mu \mathrm{m}
\end{aligned}
$$

$D_{50}=7.74 \mu \mathrm{m}$

$D_{90}=16.37 \mu \mathrm{m}$

$D_{\text {average }}=8.08 \mu \mathrm{m}$

$$
\begin{aligned}
& D_{90}=17.95 \mu \mathrm{m} \\
& D_{\text {average }}=10.21 \mu \mathrm{m}
\end{aligned}
$$
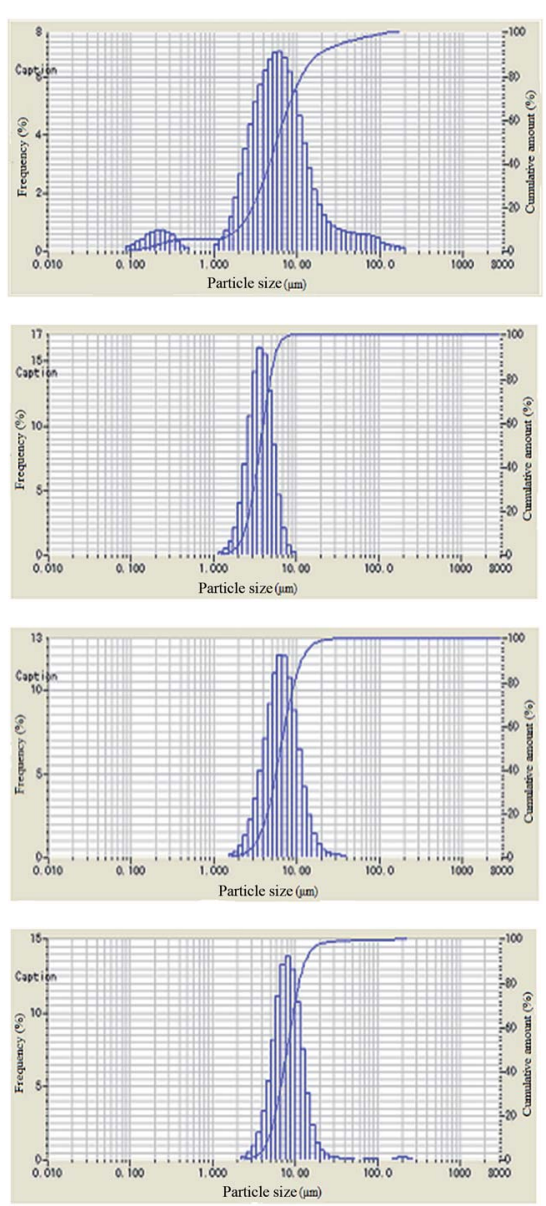

$$
\eta_{\mathrm{r}}=\frac{t}{t_{0}}
$$

$t$-the flow time of the copolymer solution, $\mathrm{s} ; t_{0}$-the flow time of $1.00 \mathrm{~mol} \mathrm{~L}^{-1} \mathrm{NaCl}, \mathrm{s} ; \eta_{\mathrm{r}}$-relative viscosity.

IR spectroscopy. Each organic substance has its own specific infrared radiation spectrum, which is determined by analyzing the compound containing the functional groups, based on the absorption peak position and strength from the infrared spectrum, which can further indicate the molecular structure. Before performing infrared analysis of DAM, necessary purification was implemented. The DAM was first dissolved in water, to which ethanol was added. The precipitates were washed several times and then dried and crushed to avoid interference from moisture and impurities. Afterwards, the purified DAM powder was blended with $\mathrm{KBr}$, compressed, and analyzed by IR spectroscopy.

\section{Results and discussion}

\section{Inhibitive properties of polyampholyte inhibitor DAM}

Particle-size distribution determination. The incorporation of organic polymer molecules could influence the interaction between bentonite particles. If clay hydration and swelling were suppressed, then the clay particles would not disperse into fine particles. Therefore, the change in particle-size distribution can be used to assess the effect of inhibitors on clay hydration. ${ }^{22}$

As shown in Table 1, the particle-size distribution curves shifted to the right and became narrow after DAM of different concentrations was added into the bentonite dispersion, demonstrating the enlargement of the bentonite particles and an effective inhibitive performance. When the concentration of polyampholyte DAM was $2 \mathrm{wt} \%$, a more obvious shift in particlesize distribution curves was observed compared to other scenarios, indicating that the optimum inhibition that DAM can achieve should be at $2 \mathrm{wt} \%$.

The evolution of median size $\left(D_{50}\right)$ and specific surface area with increasing concentration is plotted in Table 1 . When the concentration of inhibitors was low, the median size increased and the specific surface area decreased. When the concentration was higher than $2 \mathrm{wt} \%$, the median size began to decrease, and the specific surface area began to increase, indicating that relatively low concentrations were preconditions for effective DAM inhibition. 


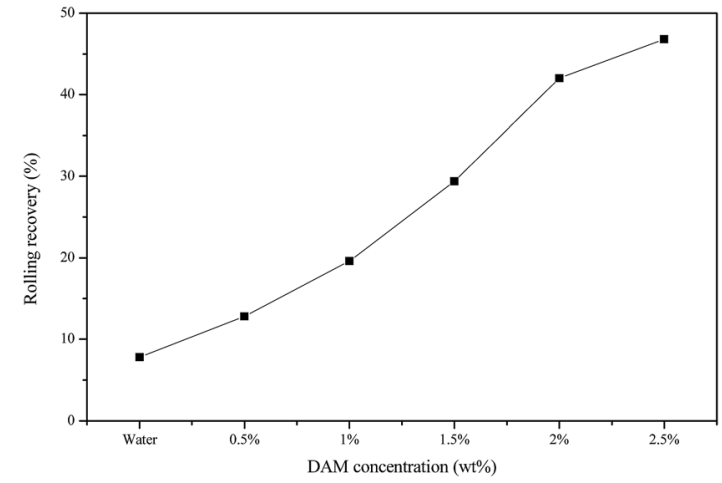

Fig. 2 Rolling recovery of different DAM concentrations (wt\%).

Table 2 Different formulas

\begin{tabular}{ll}
\hline Numbering & Formula \\
\hline $1 \#$ & Water \\
$2 \#$ & $3 \%$ KCL \\
$3 \#$ & $0.5 \%$ DAM $+3 \%$ KCL \\
$4 \#$ & $1 \%$ DAM $+3 \%$ KCL \\
$5 \#$ & $1.5 \%$ DAM $+3 \%$ KCL \\
$6 \#$ & $2 \%$ DAM $+3 \%$ KCL \\
$7 \#$ & $2.5 \%$ DAM $+3 \%$ KCL
\end{tabular}

Hot-rolling dispersion test. Hot-rolling dispersion tests were performed to evaluate the properties of inhibitor DAM. Low dispersion and high recovery indicated functional characteristics of better shale inhibition in the dispersive shale formation. The rolling recovery changed with DAM concentration, as shown in Fig. 2.

For fresh water without inhibitor, the rolling recovery was only $7.8 \%$, while the presence of DAM could improve the recovery to $12.8 \%$ when the DAM concentration reached $0.5 \mathrm{wt} \%$. The rolling recovery increased relatively sharply with an increase in DAM concentration before reaching $2 \mathrm{wt} \%$, after which the rolling recovery enhancement was relatively gentle.

The above analyses demonstrate the relatively strong ability of DAM to inhibit the dispersion of clay minerals, but the recovery was still not high because the synthesized inhibitor

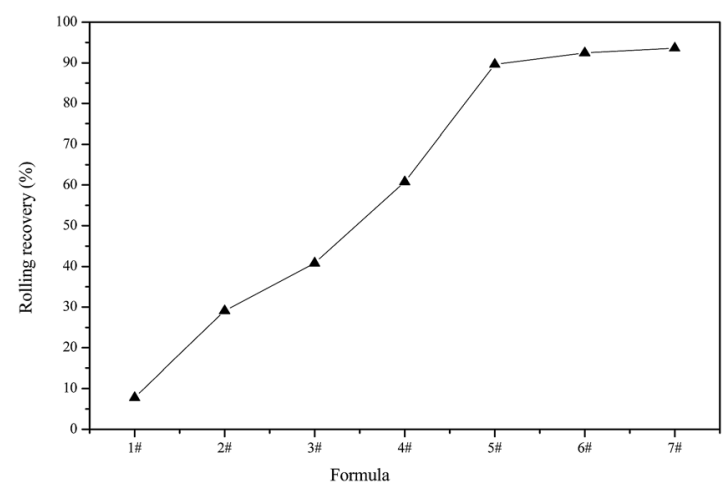

Fig. 3 Rolling recovery for different formulas.
DAM was a kind of low polymer for the highly dispersed shale cuttings. The addition of a single inhibitor failed to suppress the dispersion of clay minerals effectively, and therefore, the inhibitor DAM was added to the solution on a base of 3\% KCL. The rolling recovery results are shown in Table 2 and Fig. 3.

As shown in Table 1 and Fig. 3, the rolling recovery for 3\% KCL was $29.1 \%$. Then, with the addition of DAM, the rolling recovery increased rapidly. A solution containing $2 \mathrm{wt} \%$ DAM and $3 \mathrm{wt} \% \mathrm{KCL}$ increased the rolling recovery to $92.4 \%$, demonstrating efficient suppression. Therefore, it can be inferred that DAM had very good synergy with KCL, and greatly enhanced the suppression ability when used with other inhibitors.

Linear swelling test. Shale inhibition durability is essential in the drilling process, because failures can reoccur in previously inhibited shale formations when they are subsequently in contact with non-inhibitive fluid. ${ }^{23}$ In this experimental study, a linear swelling test was performed to investigate the inhibitive performance of DAM with different inhibitor solution concentrations.

As shown in Fig. 4, the hydration and dispersion of the NaBent pellet decreased with an increase in inhibitor concentration. Moreover, the linear swelling rate of Na-Bent under the action of DAM was lower than that under the action of a solution without inhibitor. Generally, higher inhibitor concentrations correspond to a stronger ability to inhibit clay expansion. When $3 \mathrm{wt} \% \mathrm{KCL}$ was added into the DAM solution, the swelling rate reached as low as $9.2 \%$ when the concentration of DAM was $2 \mathrm{wt} \%$. Similar results have been reported previously in terms of the strong synergistic effect to inhibit clay hydration and swelling. ${ }^{24}$

\section{Characterization of the polyampholyte inhibitor DAM}

Inherent viscosity test. The viscosity-average molecular weight of the polymer was measured using an Ubbelohde viscometer, according to the solvent and the temperature effect on $K$ and $\alpha$, which finally determined that $K=6.31 \times 10^{-3} \mathrm{~mL} \mathrm{~g}^{-1}$ and $\alpha=0.8$ at $25{ }^{\circ} \mathrm{C}$. The experimental results are shown in Table 3 .

Generally, polymers are a mixture of substances of different molecular weight, and their molecular weight distribution also has a large effect on their properties. Therefore, it is necessary

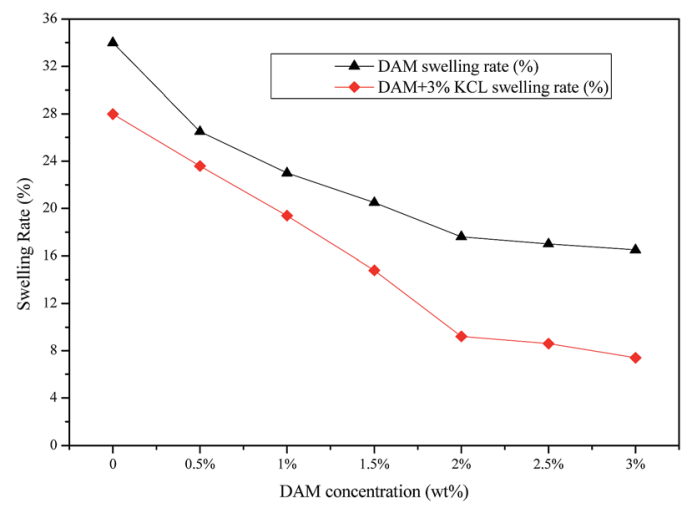

Fig. 4 Different inhibitor concentrations and their effects on linear swelling. 
Table 3 The determination parameters for DAM viscosity-average molecular weight

\begin{tabular}{ll}
\hline Parameter & Data \\
\hline$K$ & $6.31 \times 10^{-3} \mathrm{~mL} \mathrm{~g}^{-1}$ \\
$\alpha$ & 0.8 \\
{$[\eta]$} & $3.37 \mathrm{~mL} \mathrm{~g}^{-1}$ \\
$M$ & 2330
\end{tabular}

to test the molecular weight distribution of the polymer. Herein, the molecular weight distribution of DAM was determined by gel chromatography. The results are shown in Table 4 and Fig. 5.

The molecular weight distribution of DAM was narrow, and the GPC results also showed that the polydispersity of the molecular weight distribution was 1.547 . It was found that the number-average molecular weight was 1742, the weight-average molecular weight was 2695, and the Z-average molecular weight was 4300. A smaller molecular weight distribution enabled DAM to enter into the clay layers more easily so as to prevent water from entering, showing an excellent inhibitory performance.

IR spectroscopy. Infrared radiation spectroscopy is one of the most commonly used methods to determine molecular functional groups. Each organic substance has its own specific infrared spectrum, and the content of functional groups can be inferred from the infrared spectrum, based on absorption peak position and strength (Fig. 6).

As shown in Fig. 6, the absorption peak of the -OH stretching vibration appeared at $3446 \mathrm{~cm}^{-1}$, and it was strong and broad; that of the $-\mathrm{CH}_{2}-$ group stretching vibration occurred at
$2926 \mathrm{~cm}^{-1}$; that of the $-\mathrm{C}=\mathrm{O}$ bond vibration in the tertiary amide group was at $1640 \mathrm{~cm}^{-1}$; that of the $\mathrm{C}-\mathrm{N}$ bond vibration in the five-membered molecular heterocycle was at $1452 \mathrm{~cm}^{-1}$; that of the $-\mathrm{CH}_{3}$ bending vibration was at $1388 \mathrm{~cm}^{-1}$; that of the $-\mathrm{SO}_{3}$ vibration was at $1038 \mathrm{~cm}^{-1}$; and that of the $\mathrm{C}-\mathrm{S}$ bond was at $618 \mathrm{~cm}^{-1}$. The DAM was a polyampholyte inhibitor containing several anionic groups and cationic groups. The cationic groups were adsorbed on the surface of the clay particles by electrostatic interaction, and they negatively charged the surface of the clay particles. Thus, the electrostatic repulsion was reduced between the particles and the hydration shell surface, which further enhanced the aggregation between particles. At the same time, the DAM molecule contained multiple adsorbate $-\mathrm{OH}$, which could form hydrogen bonds with the alkoxy groups on the surface of the clay particles, which also resulted in multi-point adsorption to enhance its adsorption on the clay surface.

Zeta potential measurement. The zeta potential of bentonite particles in aqueous suspension was measured to probe the influence of inhibitors on the electrokinetic properties of the bentonite particles. Na-MMT particles are usually negatively charged in aqueous solution because of the isomorphic replacement of ions, as shown in Fig. 7.

The zeta potential of virgin Na-Bent was $-34.8 \mathrm{mV}$, indicating a stable dispersion. The adsorption of inhibitors changed the absolute value of the zeta potential to different degrees with an increase in concentration, while the addition of the inhibitor DAM changed the zeta potential of Na-MMT particles to different degrees. For DAM, the zeta potential changed from -34.8 to $-19 \mathrm{mV}$ with an increase in concentration. A similar result was reported by Wang et $a .^{25}$ Such behavior was

Table 4 The result of GPC

\begin{tabular}{lllllll}
\hline Name & $M_{\mathrm{n}}$ (dalton) & $M_{\mathrm{w}}$ (dalton) & $M_{\mathrm{p}}$ & $M_{\mathrm{z}}$ (dalton) & $M_{\mathrm{z}}+1$ (dalton) & Polydispersity \\
\hline Molecular weight & 1742 & 2695 & 1659 & 4300 & 6039
\end{tabular}

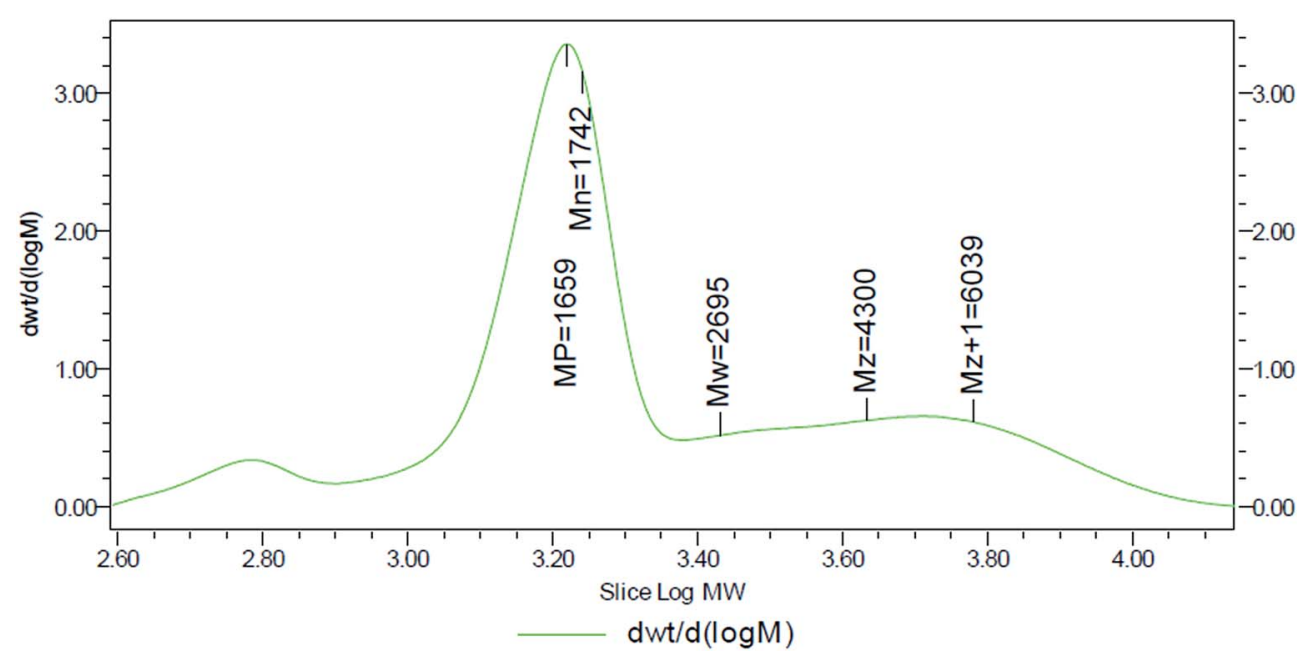

Fig. 5 The molecular weight distribution of DAM. 


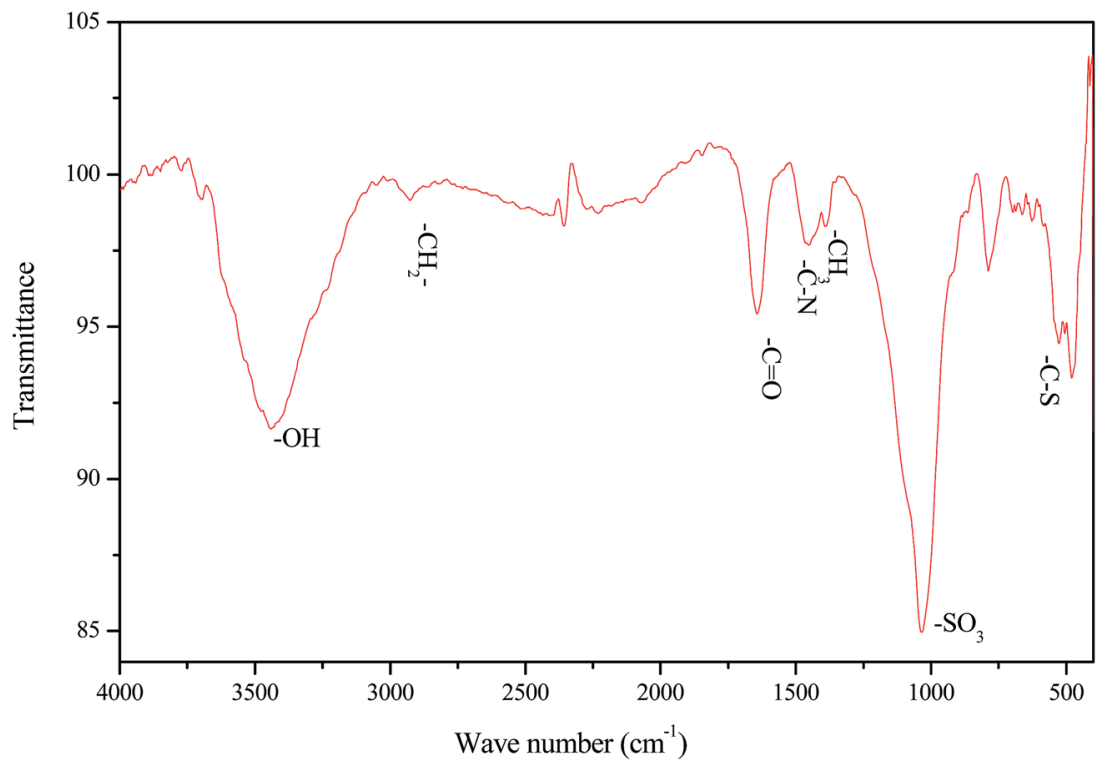

Fig. 6 Infrared spectrogram of DAM.

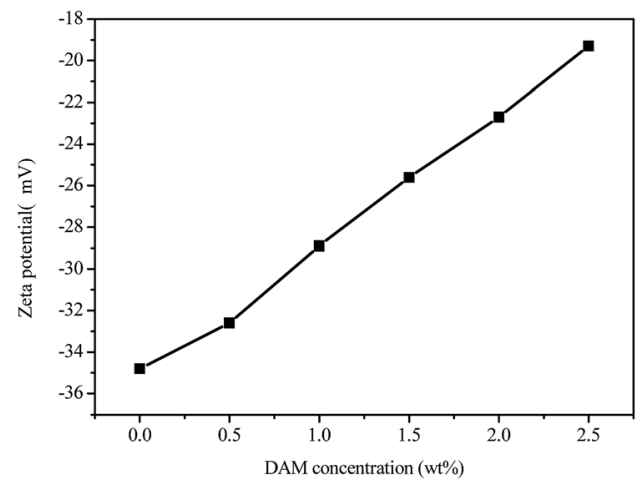

Fig. 7 Variation of zeta potential as a function of DAM concentration.

explained by a screening effect. On the one hand, in rock formations cationic groups can be adsorbed on the surface of clay minerals by the electrostatic effect, neutralizing the negative charge and providing an inhibitory effect. On the other hand, intermolecular anions and cationic groups can form a synthetic chain beam by electrostatic attraction, forming spatial reticulated structures that can coat clay particles and thus inhibit hydration and dispersion of clay minerals in the cuttings. Meanwhile, ion groups with the same charges between particles produced electrostatic repulsion, which weakened the flocculation effect. Therefore, the bentonite in the drilling fluid was moderately dispersed, which can balance the inhibition and dispersion of drilling fluids.

Amine groups would be protonated and changed into ammonium ions, which could be adsorbed onto the negatively charged sites of the clay surface by electrostatic attraction, as well as the formation of hydrogen bonds between the amine groups and the clay particles. After the adsorption, the surface of the bentonite particles was covered with a hydrophobic segment, which provided a certain shielding effect. ${ }^{26}$ The decrease in zeta potential indicated the reduced layer charge of the clay particles, resulting in a drop in the hydration repulsion of electric double layers. ${ }^{27}$ Therefore, the clay particles became less sensitive to water, and an effective hydration inhibitive performance was established. Moreover, in light of the relation between zeta potential and clay particle stability, the zeta potential value ranged from $-30 \mathrm{mV}$ to $-16 \mathrm{mV}$, which indicated the commencement of weak dispersion. ${ }^{28}$

Thermogravimetric analysis (TGA). Generally, copolymer molecules as inhibitors are susceptible to thermal degradation in a high-temperature environment, which affects the temperature resistance of the drilling fluid system. The thermal stability of DAM was tested by differential thermal-thermocouple synchronization analyzers and the test results are shown in Fig. 8.

As shown in Fig. 8, the thermal decomposition of DAM can be divided into three stages with respective corresponding temperature ranges of $40.0-105.6^{\circ} \mathrm{C}, 105.6-288.2^{\circ} \mathrm{C}$, and $288.2-409^{\circ} \mathrm{C}$.

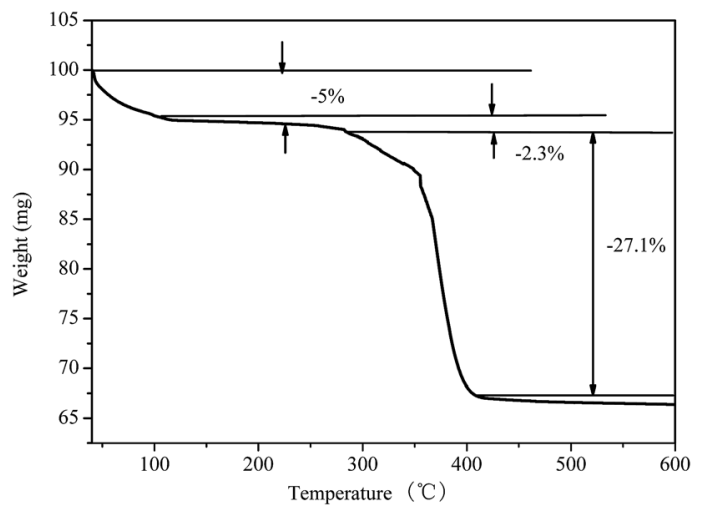

Fig. 8 Thermogravimetric analysis of DAM. 
(1) Stage 1 with temperature range of $40.0-105.6{ }^{\circ} \mathrm{C}$. During this stage, the thermal weight loss rate was slow and the weight loss was small, which can be attributed to the massive hydrophilic groups in the DAM structure, such as $-\mathrm{OH}$, as well as the easy absorption of water by the sample. Therefore, the reduction in sample quality in this temperature range was caused by the evaporation of the absorbed water..$^{29}$

(2) Stage 2 with temperature range of $105.6-288.2{ }^{\circ} \mathrm{C}$. In stage 2 , the DAM was slower and with less weight loss than in the previous stage. The DAM structure contained strong polar groups, such as - $\mathrm{CONH}-$, which enhanced the adsorption properties of the molecules and the repulsion of the polarity of water molecules, thereby enhancing the molecular inhibitory properties. The five-membered ring molecular chain formed in the synthetic polymer by DMDAAC would improve the rigidity as well as the temperature- and salt-resistance of the molecular chain. As the temperature increased, the DAM quality decreased due to the loss of combined water.

(3) Stage 3 with temperature range of $288.2-409^{\circ} \mathrm{C}$. During this stage, the weight loss decreased rapidly, indicating a faster weight loss rate and greater weight loss of DAM samples. As can be seen from Fig. 8, the quality loss of DAM during this stage was $25.2 \%$. DAM molecules began to melt and decompose, and the decomposition products evaporated after enough heat absorption.

From the analysis of the thermogravimetric curve of DAM, it can be seen that the reduction in DAM quality was lower before the ambient temperature reached $288.2{ }^{\circ} \mathrm{C}$ and without obvious thermal degradation. This is mainly due to the five-membered ring-stabilized structure of $-\mathrm{NH}_{2}-$, and its molecular structure

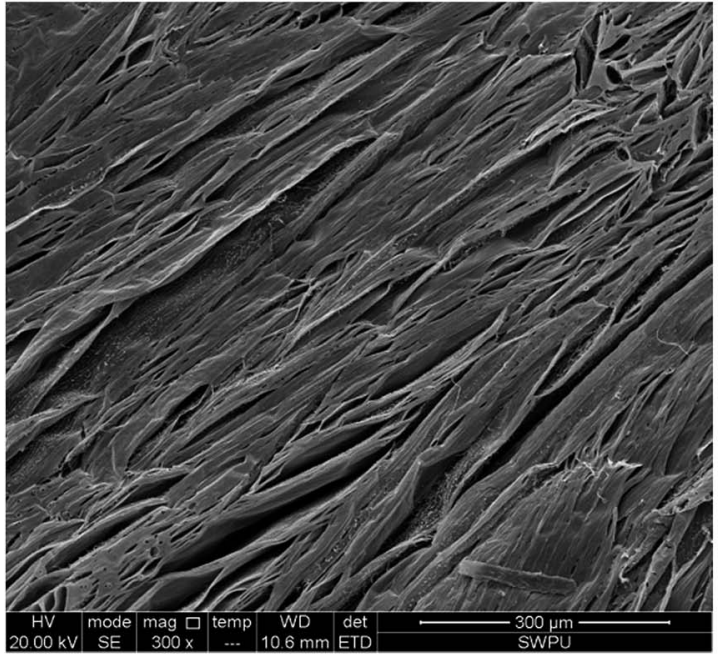

(a) 300 times

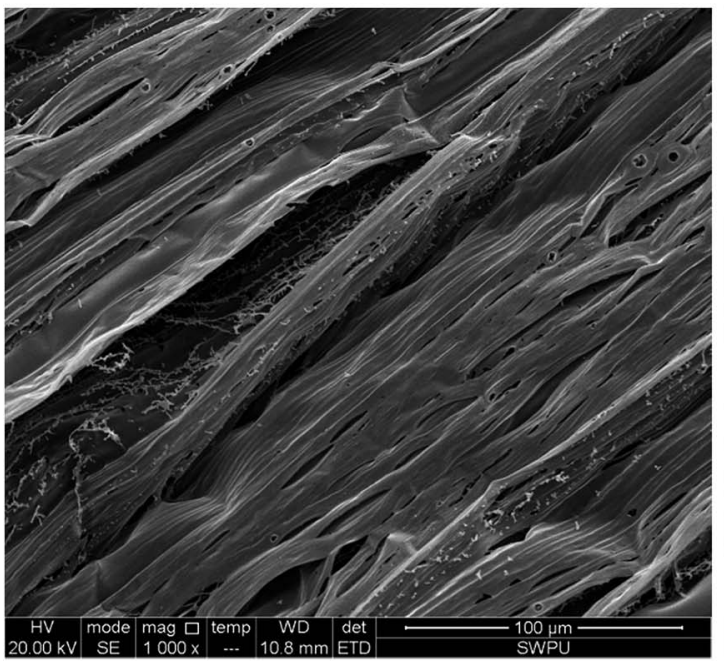

(c) 1000 times

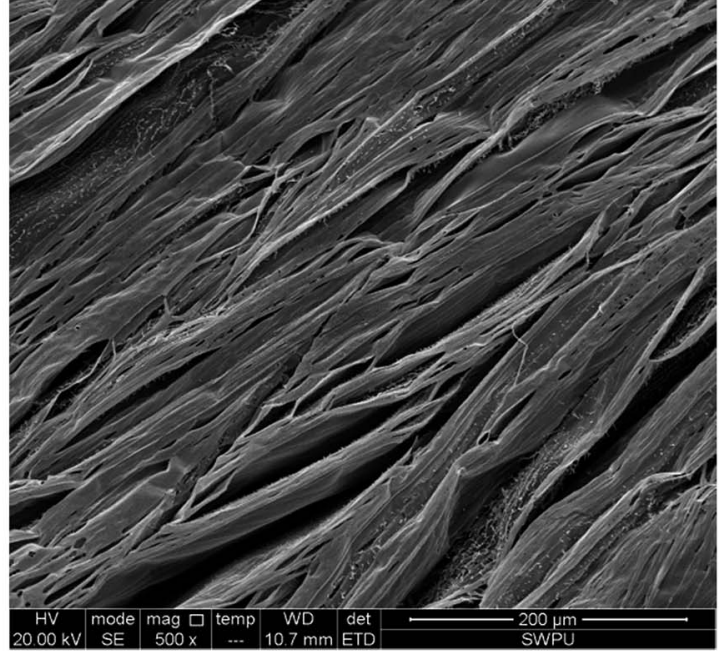

(b) 500 times

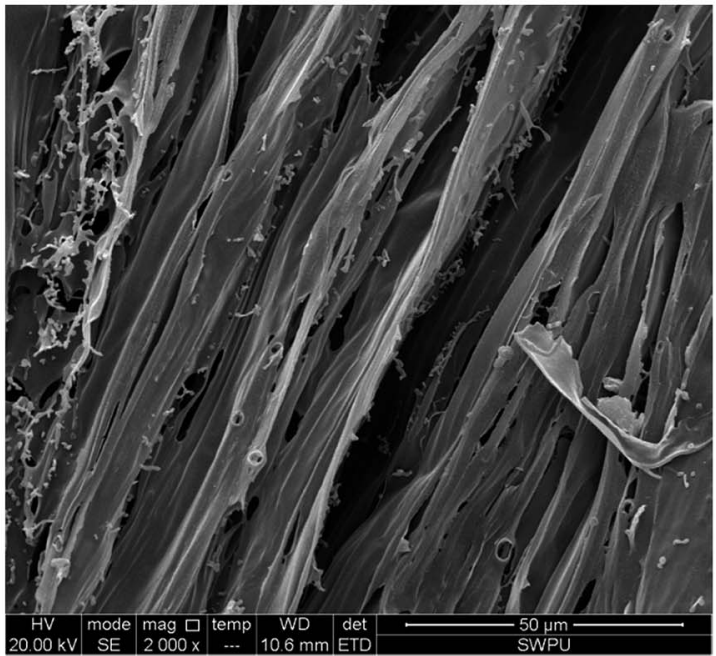

(d) 2000 times

Fig. 9 SEM images of DAM in aqueous solution. 
was not destroyed by thermal degradation at high temperatures, which means the functional groups were still effective.

DAM SEM analysis. A DAM solution with a concentration of $2.0 \%$ was prepared and placed in a sealed container for one day and frozen with liquid nitrogen, and the microstructure of DAM in deionized water was observed with SEM (Fig. 9).

DAM in aqueous solution was linear, and a proportion of the molecules were tightly connected, because the molecular structure contained both anionic and cationic groups, which were close to each other due to the action of electrostatic attraction. Some molecules were in a separate state due to the electrostatic repulsion caused by ions with the same electric properties. This linear, low molecular weight polymer is more likely to enter the layers between the clay particles, preventing water from entering and enhancing the inhibition.

Inhibitive mechanism analysis. Generally, shales can be stabilized by either chemical methods or physical methods or a combination of both. From the aspect of chemical mechanisms, ion exchange, adsorption, coating of clay particles, and modification of surface affinity toward water are the fundamental methods. ${ }^{30}$

The introduction of strongly adsorbent ammonium groups into the polymer molecular chain can not only greatly enhance the inhibiting effect of the inhibitor, but also result in the formation of five-membered ring-shaped molecular chains, which can enhance the rigidity of polymer molecules so as to improve their resistance to temperature. As $-\mathrm{SO}_{3} \mathrm{Na}$ is insensitive to the cationic affect, and the large steric hindrance of AMPS improves the rigidity of the molecular chain, the polymer can be more temperature-, salt- and calcium-resistant. Moreover, the introduction of a polar hydration group $-\mathrm{OH}$ can facilitate the adsorption of clay minerals and the formation of hydration membranes.

DAM is a linear, low molecular weight amphoteric polymer. This particular structure makes polymer chains vulnerable to association, which can coat clay particles and inhibit the hydration of clay minerals. The introduction of cationic groups into the molecular chain can lead to the ionic adsorption of clay minerals, the neutralization of some negative charges on the clay mineral surface, and weakened hydration effects on clay minerals. Such a linear, low molecular weight polymer can be more quickly and strongly adsorbed on the clay particle surface than a high molecular weight polymer. In addition, the hydrochemical membrane formed by a large number of hydration groups in the molecular chain can prevent free water molecules from contacting the clay surface, thus enhancing inhibition.

\section{Conclusions}

Under the conditions of $30 \%$ monomer concentration and a monomer ratio AMPS : DMDAAC : MP $=4: 3: 4$, the inhibitor DAM was prepared by aqueous solution polymerization at $65{ }^{\circ} \mathrm{C}$ for 7 hours. The experimental results demonstrated its lower linear expansion ratio and higher rolling recovery rate. The rolling recovery rate can reach over $90 \%$ with KCL, and the inhibition performance is excellent. Infrared radiation spectrum analysis shown that DAM contained many adsorption groups like $-\mathrm{OH}$, which could form hydrogen bonds with alkoxy groups on the surface of clay particles, and form multi-point adsorption to further enhance the absorption. The results of environmental scanning electron microscopy (SEM) identified DAM as a linear copolymer, which would lie in the clay crystal layer under the action of different chemical potentials, replace the inorganic cations between layers, neutralize the negative charge of the clay, then decrease the interlayer electrostatic repulsion, tighten the crystal layer by electrostatic action, and finally weaken the trend for layer spacing to expand. The cationic groups were adsorbed on the surface of the clay particles by electrostatic interaction, and the surface charge of the clay particles was neutralized, reducing the electrostatic repulsion between the particles and the hydration film on the surface. The multiple group synergistic action of DAM demonstrated stronger inhibitory effects, and further work to explore mechanism will continue in our laboratory.

\section{Conflicts of interest}

No conflict of interest exists in the submission of this manuscript, and manuscript is approved by all authors for publication.

\section{Acknowledgements}

The authors gratefully acknowledge the National Basic Research Program of China (973 Program, No. 2013CB228003) for financial support.

\section{References}

1 R. Steiger and P. K. Leung, Quantitative determination of the mechanical properties of shales. Paper SPE 18024, SPE Drill. Completion, 1992, 7(3), 181-185.

2 M. Khodja, J. P. Canselier, F. Bergaya, et al., Shale problems and water-based drilling fluid optimisation in the Hassi Messaoud Algerian oil field, Appl. Clay Sci., 2010, 49(4), 383-393.

3 M. Josh, L. Esteban, C. D. Piane, et al., Laboratory characterisation of shale properties, J. Pet. Sci. Eng., 2012, 88-89(2), 107-124.

4 A. Gueciouer, A. Benmounah, H. Sekkiou, et al., Valorization of $\mathrm{KCl} / \mathrm{PHPA}$ system of water-based drilling fluid in presence of reactive clay: Application on Algerian field, Appl. Clay Sci., 2017, 146, 291-296.

5 M. Khodja, J. P. Canselier, F. Bergaya, et al., Shale problems and water-based drilling fluid optimisation in the Hassi Messaoud Algerian oil field, Appl. Clay Sci., 2010, 49(4), 383-393.

6 Y. Qu, X. Lai, L. Zou, et al., Polyoxyalkyleneamine as shale inhibitor in water-based drilling fluids, Appl. Clay Sci., 2009, 44(3-4), 265-268.

7 D. Obrien and M. E. Chenevert, Stabilizing Sensitive Shales With Inhibited, Potassium-Based Drilling Fluids, J. Pet. Technol., 1973, 25(9), 1089-1100. 
8 G. Odriozola and J. F. Aguilar, Stability of K-Montmorillonite Hydrates: Hybrid MC Simulations, J. Chem. Theory Comput., 2005, 1(6), 1211.

9 W. Du, X. Pu, J. Sun, et al., Synthesis and evaluation of a novel monomeric amine as sodium montmorillonite swelling inhibitor, Adsorpt. Sci. Technol., 2017, 026361741771385 .

10 H. Zhong, Z. Qiu, W. Huang, et al., Shale inhibitive properties of polyether diamine in water-based drilling fluid, J. Pet. Sci. Eng., 2011, 78(2), 510-515.

11 A. Patel, Design and Development of Quaternary Amine Compounds: Shale Inhibition with Improved Environmental Profile, 2009.

12 S. Gou, T. Yin, Q. Xia, et al., Biodegradable polyethylene glycol-based ionic liquids for effective inhibition of shale hydration, RSC Adv., 2015, 5(41), 32064-32071.

13 H. Zhong, Z. Qiu, W. Huang, et al., Shale inhibitive properties of polyether diamine in water-based drilling fluid, J. Pet. Sci. Eng., 2011, 78(2), 510-515.

14 Y. Xuan, G. Jiang, Y. Li, et al., Biodegradable oligo (poly-Llysine) as a high-performance hydration inhibitor for shale, RSC Adv., 2015, 5(103), 84947-84958.

15 J. R. Bruton and H. C. Mclaurine, Modified Poly-Amino Acid Hydration Suppressant Proves Successful in Controlling Reactive Shales, 1993.

16 M. Scholl, T. Q. Nguyen, B. Bruchmann and H. A. Klok, J. Polym. Sci., Part A: Polym. Chem., 2007, 45, 5494-5508.

17 S. W. Fox and K. Harada, Arch. Biochem. Biophys., 1960, 86, 281-285.

18 W. Shan, S. Tao, F. Fu, W. Yue and Z. Zhao, Research on the drilling fluid technology for high temperature over $240{ }^{\circ} \mathrm{C}$, Procedia Eng., 2014, 73, 218-229.

19 H. Mao, Z. Qiu, Z. Shen and W. Huang, Hydrophobic associated polymer based silica nanoparticles composite with core-shell structure as a filtrate reducer for drilling fluid at ultra-high temperature, J. Pet. Sci. Eng., 2015, 129, 1-14.

20 M. Khodja, J. P. Canselier, F. Bergaya, K. Fourar, M. Khodja, N. Cohaut and A. Benmounah, Shale problems and water- based drilling fluid optimization in the Hassi Messaoud Algerian oil field, Appl. Clay Sci., 2010, 49, 383-393.

21 A. Patel, E. Stamatakis, S. Young and S. Cliffe, Designing for the future: a review of the design, development and testing of a novel, inhibitive water-based drilling fluid, in AADE-02DFWM-HO-33, AADE 2002 Technical Conference, Texas, 2-3 April 2002.

22 S. Liu, X. Mo, C. Zhang, D. Sun and C. Mu, Swelling inhibition by polyglycols in montmorillonite dispersions, $J$. Dispersion Sci. Technol., 2004, 25, 63-66.

23 M. Amanullah, M. Al-Arfaj and A. Al-Ansari, Method for prediction of inhibition durability index of shale inhibitors and inhibitive drilling mud systems, US 9164018, 2015.

24 P. I. Reid, B. Dolan and S. Cliffe, Mechanism of shale inhibition by polyols in water based drilling fluids, SPE 28960, SPE International symposium on Oilfield Chemistry, San Antonio, 14-17 Feb. 1995.

25 L. Wang, S. Liu, T. Wang and D. Sun, Effect of poly(oxypropylene)diamine adsorption on hydration and dispersion of montmorillonite particles in aqueous solution, Colloids Surf., A, 2011, 381(1-3), 41-47.

26 J. J. Lin, Y. M. Chen and M. H. Yu, Hydrogen-bond driven intercalation of synthetic fluorinated mica by poly(oxypropylene)-amidoamine salts, Colloids Surf., A, 2007, 302(1-3), 162-167.

27 K. K. Mohan and H. S. Fogler, Effect of $\mathrm{pH}$ and layer charge on formation damage in porous media containing swelling clays, Langmuir, 1997, 13, 2863-2872.

28 A. J. Ritter and R. Geraut, New optimization drilling fluid programs for reactive shale formations, SPE 14247, 60th Annual Technical Conference and Exhibition of the Society of Petroleum Engineers, Las Vegas, 22-25 Sept. 1985.

29 L. Marçal, E. H. De Faria, E. J. Nassar, R. Trujillano, N. Martín, M. A. Vicente, V. Rives, A. Gil, S. A. Korili and K. J. Ciuffi, Organically modified saponites: SAXS study of swelling and application in Caffeine removal, ACS Appl. Mater. Interfaces, 2015, 7, 10853-10862.

30 A. Zaltoun and N. Berton, Stabilization of montmorillonite clay in porous media by high-molecular-weight polymers, SPE Prod. Eng., 1992, 7(2), 160-166. 